\title{
Research on "load- grid - source" collaborative optimization Considering incremental load caused by electric power alternative
}

\author{
Yahui Wua , Bingqing $\mathrm{Guo}^{\mathrm{b}}$, Limin Jiang ${ }^{\mathrm{c}}$, Guixiong $\mathrm{He}^{\mathrm{d}}$, Kaicheng Liu ${ }^{\mathrm{e}}$ \\ China Electric Power Research Institute, Beijing 100192, China \\ awuyahui1018@163.com, bbq_guo@epri.sgcc.com.cn, cjianglm@epri.sgcc.com.cn, \\ dheguixiong@epri.sgcc.com.cn, eliukaicheng@epri.sgcc.com.cn
}

Keywords: electric power alternative, incremental load, "load- grid - source" collaborative optimizat ion.

\begin{abstract}
With the development of electric power alternative, the incremental flexible load in power grid plays a more important role. This paper from the perspective of sociology, consider the profit of the accommodation of new energy increment power generation and load adjustment,and the cost of the generator increment power generation and the grid increment loss, Optimize the amount of incremental flexible load adjustment and the generator increment power generation, Establish a "load-net-source" collaborative optimization mathematical model with the aim of maximizing social benefits, The feasibility of the model is verified by using the IEEE30 node example, Using the successive linearization algorithm to solve the problem, and through the IEEE30 node example to verify the rationality and effectiveness of the model.
\end{abstract}

\section{Introduction}

It is the world difficult problem for large-scale new energy grid-connected and digestion because the randomness and volatility of new energy sources [1]. The implementation of "two alternative" strategies provides new ideas for new energy consumption. Document [2] defines the quantification calculation of the electric energy substitution implements the electric energy substitution quantity, establishes its (human impact of population affluence and Technology) IPAT model, and analyzes the potential of electric energy substitution by constructing many scenes; Document [3]propose a project to use wind power curtailment to winter heating for the problem of wind power curtailment in Zhangjiakou, through the establishment of calculation model of electric load, wind power accommodation and environmental benefit calculation model, mainly from the social and environmental benefits and the impact on the power system, verify the feasibility of the scheme.

[4][5][6]consider the flexible response of load which through changing the duration and size of load application to response power operator scheduling requirements which obtain certain economic benefits, the future smart grid will accommodate a huge amount of flexible load. As an important resource for power generation scheduling, flexible load has the advantages of peak load shifting, stabilizing the fluctuation of new energy output and providing ancillary services for the grid, which enriching the power grid dispatching mode. Document [4]pointed out that a large amount of incremental flexible load is generated with the advance of electric energy substitution, power energy alternative increment loads have strong polymerization that can improve the control efficiency through the distribution of cluster, as virtual storage or virtual generator the load aggregators can participate in the grid-interactive to response dispatching instruction and provide ancillary services for power grid; in paper[8], a multi-time scale response framework is proposed, and a multi-time scale response strategy of flexible load participation is proposed by establishing wind power output uncertainty model, load agent decision making and scheduling control center decision model; In the literature [9], the overall framework of "source-network-charge" interaction is proposed, and it is pointed out that the future flexible power system will show a variety of interactive modes such as complementary sources, source network coordination, grid interaction and source interaction; In the literature [10], by analyzing the adjustability of the high energy-carrying enterprises, it proves the 
feasibility of the new energy consumption in the intelligent park, and combines the mixed energy and energy storage modeling in the intelligent park, the power and load are jointly scheduled which improve the local energy consumption of new energy.

However, the existing research is generally considered a separate flexible load, not considering the impact of just load, with the release of the electricity market side, more and more power users who contains the just loads, the electricity loads and the incentive loads to participate in the electricity market which rich the main body of the electricity market. Based on the "two alternatives" strategy, this paper takes the basic load as the starting point, and then deduces the new flexible load value of the power grid, and establishes the "load - grid - source" collaborative optimization model based on the new flexible load that provide a theoretical basis for large-scale direct purchase of electricity and bilateral transactions in China's electricity market.

\section{Theoretical Model of the Power Grid Incremental Power Loss}

The incremental network loss caused by the incremental load of the power network can be calculated as follows:

$$
\begin{aligned}
& \Delta P_{\text {loss }}=P_{\text {load }} \cdot P_{\text {load }}= \\
& \sum_{j=1}^{n} \sum_{k=1}^{n} U_{j}^{*} U_{k}^{*} G_{j k} \cos \delta_{i j}^{*}-\sum_{j=1}^{n} \sum_{k=1}^{n} U_{j} U_{k} G_{j k} \cos \delta_{j k}
\end{aligned}
$$

Consider the incremental load of the power balance equation:

$$
\sum_{i=1}^{n} \Delta P_{G i}-\sum_{i=1}^{n} \Delta P_{l i}-\Delta P_{\text {loss }}=0
$$

Where $\Delta P_{G i}$ is the output change of the generator, $\Delta P_{l i}$ incremental load change and $\Delta P_{\text {loss }}$ is incremental power loss.

Compared with the stock load, because the incremental load value is relatively small, if the whole system for a new power flow calculation, the operation speed will be greatly reduced, where we use the sensitivity analysis method, not only improve the calculate speed, but also meet the scheduling staff needs and provide theoretical support for the scheduling staff.

The nodal equilibrium equation can be expressed as:

$$
\begin{aligned}
& P_{i}=U_{i} \sum_{j=1}^{n} U_{j}\left(G_{i j} \cos \delta_{i j}+B_{i j} \sin \delta_{i j}\right) \\
& Q_{i}=U_{i} \sum_{j=1}^{n} U_{j}\left(G_{i j} \sin \delta_{i j}-B_{i j} \cos \delta_{i j}\right)
\end{aligned}
$$

The branch power equation as follows:

$$
\begin{aligned}
& P_{i j}=U_{i}^{2} g_{i j}-U_{i} U_{j}\left(g_{i j} \cos \delta_{i j}+b_{i j} \sin \delta_{i j}\right) \\
& Q_{i j}=-U_{i}^{2}\left(b_{i j}+b_{i o}\right)+U_{i} U_{j}\left(b_{i j} \cos \delta_{i j}-g_{i j} \sin \delta_{i j}\right)
\end{aligned}
$$

The power grid loss can be presented as follow:

$$
P_{\text {loss }}=\sum_{j=1}^{n} \sum_{k=1}^{n} U_{j} U_{k} G_{j k} \cos \delta_{j k}
$$

From Eqs. (5) and (6) we get the Sensitivity Analysis of Branch Power to State Variables as follow:

$$
\begin{aligned}
& {\left[\begin{array}{l}
\Delta P_{i j} \\
\Delta Q_{i j}
\end{array}\right]=\left[\begin{array}{ll}
2 u_{i} g_{i j}-u_{j}\left(g_{i j} \cos \delta_{i j}+b_{i j} \sin \delta_{i j}\right) & ,-u_{i}\left(g_{i j} \cos \delta_{i j}+b_{i j} \sin \delta_{i j}\right) \\
-2 u_{i}\left(b_{i j}+b_{i j}\right)+u_{j}\left(b_{i j} \cos \delta_{i j}-g_{i j} \sin \delta_{i j}\right), u_{i}\left(b_{i j} \cos \delta_{i j}-g_{i j} \sin \delta_{i j}\right)
\end{array}\right]\left[\begin{array}{l}
\Delta u_{i} \\
\Delta u_{j}
\end{array}\right]} \\
& +\left[\begin{array}{l}
u_{i} u_{j}\left(g_{i j} \sin \delta_{i j}-b_{i j} \cos \delta_{\delta_{i j}}\right), u_{i} u_{j}\left(b_{i j} \cos \delta_{i j}-g_{i j} \sin \delta_{i j}\right) \\
u u_{i} u_{j}\left(-b_{i j} \sin \delta_{\delta_{i j}}-g_{i j} \cos \delta_{i j}\right), u_{i} u_{j}\left(b_{i j} \sin \delta_{i j}+g_{i j} \cos \delta_{i j}\right)
\end{array}\right]\left[\begin{array}{l}
\Delta \delta_{i} \\
\Delta \delta_{j}
\end{array}\right] \\
& =\mathrm{A}\left[\begin{array}{l}
\Delta u_{i} \\
\Delta u_{j}
\end{array}\right]+B\left[\begin{array}{l}
\Delta \delta_{i} \\
\Delta \delta_{j}
\end{array}\right]
\end{aligned}
$$

Where the matrix A and B are constant matrices.

From (7) we can get the Sensitivity analysis of state loss to state variables as follow:

$\Delta P_{\text {loss }}=\left[\frac{\partial P_{\text {loss }}}{\partial \delta^{T}}, \frac{\partial P_{\text {loss }}}{\partial U^{T}}\right]\left[\begin{array}{l}\Delta \delta \\ \Delta U\end{array}\right]$

Sensitivity Analysis of Node Injection Power to State Variables can be writted as (10) 
$\left[\begin{array}{c}\Delta P \\ \Delta Q\end{array}\right]=\left[\begin{array}{l}\frac{\partial P}{\partial \delta^{T}}, \frac{\partial P}{\partial U^{T}} \\ \frac{\partial Q}{\partial \delta^{T}}, \frac{\partial Q}{\partial U^{T}}\end{array}\right]\left[\begin{array}{l}\Delta \delta \\ \Delta U\end{array}\right]=J\left[\begin{array}{l}\Delta \delta \\ \Delta U\end{array}\right]$

From (10) we get

$\left[\begin{array}{l}\Delta P_{G}-\Delta P_{L} \\ \Delta Q_{G}-\Delta Q_{L}\end{array}\right]=\left[\begin{array}{l}\frac{\partial P}{\partial \delta^{T}}, \frac{\partial P}{\partial U^{T}} \\ \frac{\partial Q}{\partial \delta^{T}}, \frac{\partial Q}{\partial U^{T}}\end{array}\right]\left[\begin{array}{c}\Delta \delta \\ \Delta U\end{array}\right]=J\left[\begin{array}{c}\Delta \delta \\ \Delta U\end{array}\right]$

Form (10) and (11) we can get the sensitivity of load to generator output as follow:

$$
\begin{aligned}
{\left[\begin{array}{c}
\Delta \delta \\
\Delta U
\end{array}\right] } & =J^{-1}\left[\begin{array}{c}
\Delta P \\
\Delta Q
\end{array}\right]=\left[\begin{array}{ll}
\frac{\partial P}{\partial \delta^{T}}, \frac{\partial P}{\partial U^{T}} \\
\frac{\partial Q}{\partial \delta^{T}}, \frac{\partial Q}{\partial U^{T}}
\end{array}\right]^{-1}\left[\begin{array}{c}
\Delta P \\
\Delta Q
\end{array}\right] \\
& =\left[\begin{array}{ll}
\frac{\partial P}{\partial \delta^{T}}, \frac{\partial P}{\partial U^{T}} \\
\frac{\partial Q}{\partial \delta^{T}}, \frac{\partial Q}{\partial U^{T}}
\end{array}\right]^{-1}\left[\begin{array}{l}
\Delta P_{G}-\Delta P_{L} \\
\Delta Q_{G}-\Delta Q_{L}
\end{array}\right]
\end{aligned}
$$

In conclusion, we get

$$
\begin{aligned}
\Delta P_{\text {loss }} & =\left[\frac{\partial P_{\text {loss }}}{\partial \delta^{T}}, \frac{\partial P_{\text {loss }}}{\partial U^{T}}\right]\left[\begin{array}{l}
\Delta \delta \\
\Delta U
\end{array}\right] \\
& =\left[\frac{\partial P_{\text {loss }}}{\partial \delta^{T}}, \frac{\partial P_{\text {loss }}}{\partial U^{T}}\right]\left[\begin{array}{l}
\frac{\partial P}{\partial \delta^{T}}, \frac{\partial P}{\partial U^{T}} \\
\frac{\partial Q}{\partial \delta^{T}}, \frac{\partial Q}{\partial U^{T}}
\end{array}\right]^{-1}\left[\begin{array}{l}
\Delta P_{G}-\Delta P_{L} \\
\Delta Q_{G}-\Delta Q_{L}
\end{array}\right]
\end{aligned}
$$

\section{Generator Marginal Cost and Load Marginal Revenue Model}

\subsection{Simplification Model of Generator Marginal Cost}

Power generation costs are closely related to power generation, where binomial functions are used to represent generators' active cost functions, i. e.:

$$
F_{G i}=A_{G i} P_{G i}^{2}+B_{G i} P_{G i}+C_{G i}
$$

Then we get the generator marginal cost as ():

$$
M C_{G i}=\frac{d F_{G i}}{d P_{G i}}=2 A_{G i} P_{G i}+B_{G i}
$$

Combined with the actual situation, this paper uses the simplified piecewise linear model shown in Fig. 1 to represent the marginal cost of different units.

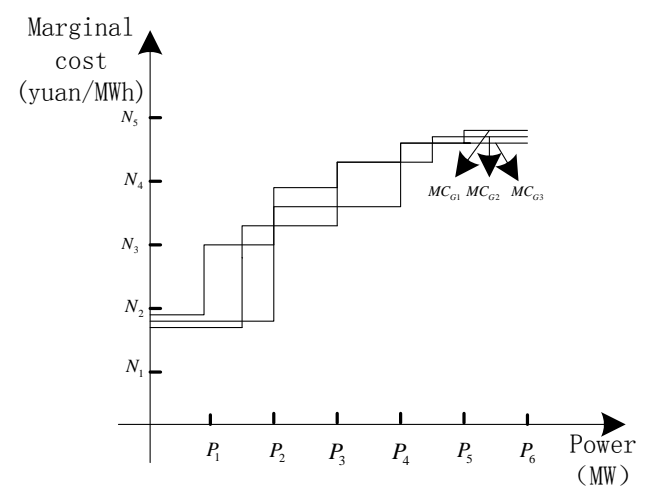

Fig. 1 The marginal cost reduction model of the unit

\subsection{The Marginal Revenue model of the Load Participates in the Regulation}

After the reform of the electricity sales side, the main body of the electricity market is gradually rich, Power users are divided into options and control users depending on whether there is an option at power purchase. The power users have the option to choose purchasing through electricity retail 
company agent, or with the power generation enterprises to conduct direct transactions. The large industrial users of Power users whose electricity costs accounted for a large proportion in the total cost of production, and its production decision-making will be more sensitive to the price, while the user has a certain adjustable load, making large industrial users to become a very good power grid scheduling Resources that can obtain a certain amount of additional benefits in response to the Power dispatching instructions, such as with less electricity to obtain electricity or expand the reproduction to obtain more profits.

For the flexible incremental load generated by the electric power alternative, the gain is obtained by providing the auxiliary service by means of the virtual accumulator or the virtual generator in the form of the loader. To the electric heating load (including energy storage link), for example, the aggregator can respond to the demand of the grid by optimizing the start-stop of the electric heating load and responding to peak shaving requirements of power grid; When the load is low and the wind power is large, the electric energy is converted into heat (cold) by means of a heat storage (cold) device, and heat (cold) is released to its users during the peak period. Responding to fill the valley and consumption of new energy requirements.

\section{4. "Load - Grid - Source" Collaborative and Successive Optimization Mathematical Models}

Based on the optimal power flow technology, digging the regulation potential of incremental load with adjustable and interruptible characteristic, increasing the consumption of new energy consumption under the premise of system security constraints, At the same time, reasonable arrangements for conventional unit of incremental output caused by the incremental load, to maximize the benefits of the whole society, including new energy incremental power consumption income, load adjustment benefits, the costs of conventional unit incremental output and incremental loss caused by incremental load.

New energy incremental power consumption income can be write as follow:

$c_{G 1}\left[c_{G 1,1}, c_{G 1,2} \cdots c_{G 1, n}\right]$

For the marginal cost of generating the incremental output of conventional units, the simplified model used in this paper is adopted, i. e.:

$$
c_{G 2}\left[c_{G 2,11}, c_{G 2,12} \cdots ; c_{G 2,21}, c_{G 2,22} \cdots ; c_{G 2, n 1}, c_{G 2, n 2} \cdots\right]
$$

The marginal revenue of load adjustment for different users and the marginal cost of loss for different voltage levels can be expresses as:

$$
c_{L}\left[c_{L 1}, c_{L 2}, \cdots, c_{L n}\right] ; c_{N}\left[c_{N 1}, c_{N 2}, \cdots, c_{N n}\right]
$$

From (16), (17), (18) we can get the objective function of maximizing social benefits as follow:

$$
\begin{aligned}
\max \mathrm{C}= & \left(\mathrm{c}_{G 1}-c_{G 2}\right)\left(\Delta P_{G 1}, \Delta P_{G 2}, \cdots\right)+c_{L}\left(\Delta P_{L 1}, \Delta P_{L 2}, \cdots\right) \\
& -c_{N}\left(\Delta P_{\text {loss }}, \Delta P_{\text {loss } 2}, \cdots\right)
\end{aligned}
$$

The constraints are as follows:

(1) Power balance constraint can be written as follow:

$$
\sum_{i=1}^{N} P_{i}(t)+\sum_{i=1}^{N_{W}} P_{W i}(t)+\sum_{i=1}^{N_{P V}} P_{P V i}(t)=\sum_{i=1}^{N_{l}} P_{l i}(t)
$$

Where ${ }^{P_{i}(t)} \mathrm{c}^{P_{W i}(t)}, P_{P V i}(t), P_{l i}(t)$ is conventional power, wind power, photovoltaic and load power for the point time of $\mathrm{t}$ respectively.

(2) Unit output constraints can be written as follow:

$$
\begin{aligned}
& P_{i \text { min }} \leq P_{i} \leq P_{i \text { max }} \\
& P_{W i \text { min }} \leq P_{W i} \leq P_{W i \max } \\
& P_{P V i \text { min }} \leq P_{P V i} \leq P_{P V i \max }
\end{aligned}
$$

(3) Unit positive and negative rotation reserve constraints can be writen as follow: 


$$
\begin{aligned}
& \sum_{i=1}^{N} P_{i \max }(t) \geq\left(\sum_{i=1}^{N_{t}} P_{l i}(t)\right) \cdot(1+L \%)+\left(\sum_{i=1}^{N_{W}} P_{W i}(t)\right) \cdot u_{w} \%+\left(\sum_{i=1}^{N_{p V}} P_{P V i}(t)\right) \cdot u_{p v} \% \\
& \sum_{i=1}^{N} P_{i \min }(t) \leq\left(\sum_{i=1}^{N_{l}} P_{l i}(t)\right) \cdot(1-L \%)-\left(P_{W}(t)-\sum_{i=1}^{N_{W}} P_{W i}(t)\right) \cdot d_{w} \%+\left(P_{P V}(t)-\sum_{i=1}^{N_{P V}} P_{P V i}(t)\right) \cdot d_{p v} \%
\end{aligned}
$$

Where, $P_{i \max }(t), P_{i \min }(t)$ is the maximum and minimum technical output of the conventional unit respectively, $L \%$ is positive and negative demand factor for the total load of the system; $u_{w} \%, u_{p v} \%$ 分is positive and negative demand factor for the prediction error of wind power and photovoltaic respectively; $P_{W}(t), P_{P V}(t)$ is the total installed capacity of wind power and photovoltaic respectively.

(4) Conventional unit climbing rate constraint can be written as follow:

$-d_{i} T_{t} \leq P_{i}(t)-P_{i}(t-1) \leq u_{i} T_{t}$

Where $d_{i}, u_{i}$ is decline and rise climb rate respectively; $T_{t}$ is scheduling time scales.

\section{Model solving}

Aiming at the problem of "load - grid - source" collaborative and successive optimization in this paper, we choose a time segment to optimization the increment change of the load and source, and consider the influence of the network loss under different strategies to maximize the benefits of the whole society. In this paper, particle swarm optimization (PSO) is used and load and source variation are taken as individuals in the algorithm by using the real matrix. The concrete form is as follows:

Variation of load adjustment and generator output can be expressed as follows:

$$
X_{l}=\left[\Delta P_{l 1}, \Delta P_{l 2} \cdots \Delta P_{l n}\right]^{T}=\left[\mathrm{x}_{l 1}, \mathrm{x}_{l 2}, \cdots, \mathrm{x}_{l n}\right]^{T} X_{G}=\left[\Delta P_{G 1}, \Delta P_{G 2} \cdots \Delta P_{G n}\right]^{T}=\left[\mathrm{x}_{G 1}, \mathrm{x}_{G 2}, \cdots, \mathrm{x}_{G n}\right]^{T}
$$

Where $U$ is Boolean variable column matrix to determine the adjustable load node, that is, by calculating $U^{T} X_{l}$ to determine the total amount of load involved in regulation. In the step-by-step optimization process, the objective function is used to calculate the current marginal cost according to the running state of the unit. To determine the priority order of a certain power interval for generators, when the marginal cost of different units is equal, it is incremental transmission losses that determine the priority of the unit to increase or decrease the order of output. Specific optimization steps shown in Figure 2.

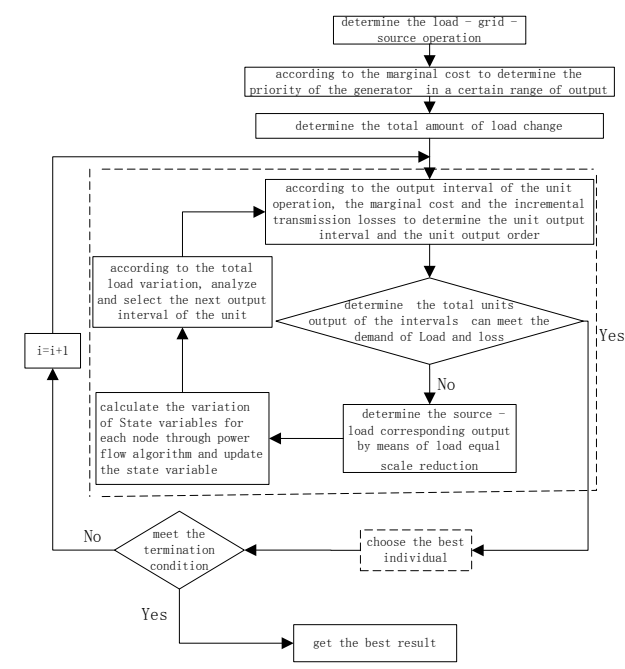

Fig. 2 The flow chart of" load-grid-source" collaborative optimization.

As shown in Fig2, the contents of the dashed line indicates the step-by-step optimization process, and the state variables are updated step by stepwise: $U^{\prime}=U+\Delta U, \delta=\delta+\Delta \delta$, In the step-by-step op timization process, through load equal scale reduction in each iteration process in order to achieve th e gradual and accurate calculation of the loss, the specific calculation steps shown in Fig. 3 . Calcula te by stepwise linear optimization $\Delta P_{l}=\Delta P_{l, 1}+\Delta P_{l, 2}+\cdots, \Delta P_{\text {loss }}=\Delta P_{\text {loss } 1}+\Delta P_{\text {los } 2,2}+\cdots$ and $\Delta P_{G}=\Delta P_{G, 1}+\Delta P_{G, 2}+\cdots$ to achieve the optimal solution of the objective function. 


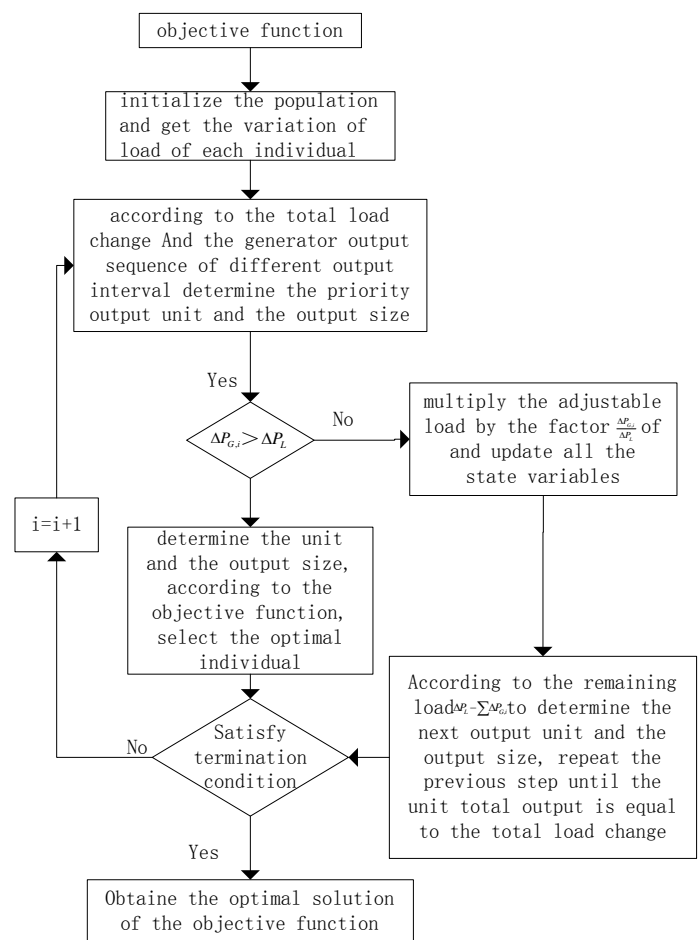

Fig. 3 Proportional load reduction calculation flow chart.

\section{Case study and analysis}

References are cited in the text just by square brackets [1]. (If square brackets are not available, slashes may be used instead, e. g. /2/.) Two or more references at a time may be put in one set of

In order to validate the validity of the model and algorithm, this paper takes IEEE30 node system as an example. Figure 4 for the IEEE30 node system structure (reference value of $S=100 \mathrm{MVA}$ ), this system contains six units, 21 load nodes, if the system has five adjustable load nodes. In this paper, renumbering of the nodes is that first PQ nodes, the PV nodes again, and the final balance node. The standby demand factor of the wind turbine is 0.15 , the specific parameters of the line and transformer, the load data and the initial output data of the power are described in [11].

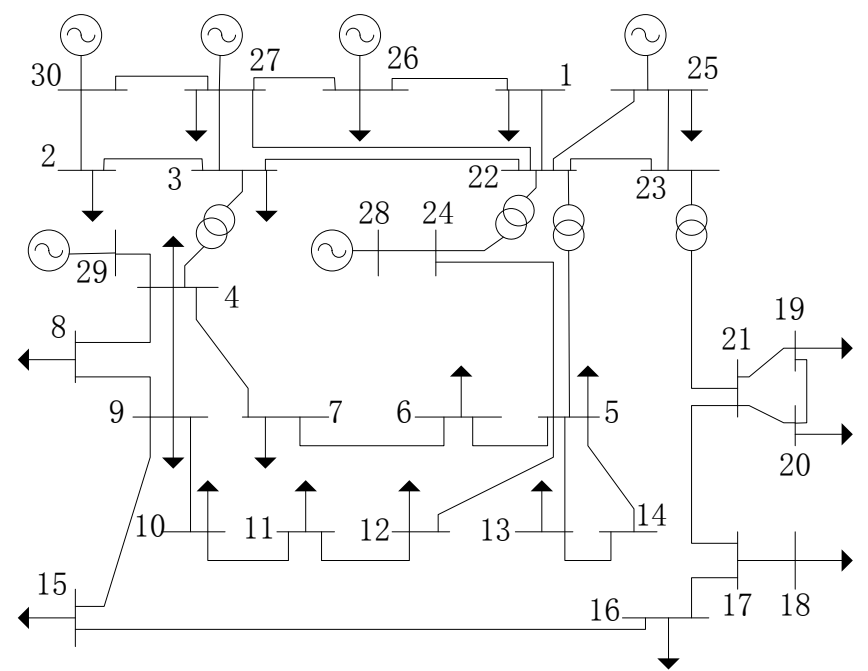

Fig. 4 Network configuration of IEEE 30-bus system.

In this paper, the load 1, 13, 25, 26 and 27 as adjustable loads and the specific adjustment interval and marginal revenue as shown in Table 1, and the loss cost is 200yuan/ MWh. 
Table 1. The adjustment interval and adjust marginal benefit of each node load.

\begin{tabular}{cccc}
\hline node & $\begin{array}{c}\text { Load status } \\
(\mathrm{MW})\end{array}$ & Regulation interval & marginal revenue (yuan/MWh) \\
\hline 1 & 22.8 & {$[20,25]$} & 280 \\
13 & 17.5 & {$[15,20]$} & 290 \\
25 & 30 & {$[20,40]$} & 300 \\
26 & 94.2 & {$[90,100]$} & 350 \\
27 & 21.7 & {$[20,25]$} & 270 \\
\hline
\end{tabular}

The marginal cost of node 27 (60MW), node 26 (30MW), node 28, 29 (25MW) and node 30 $(200 \mathrm{MW})$ in different output intervals is shown in Figure 5, where $\mathrm{x}$ points indicate the initial operating status. Node $60(60 \mathrm{MW})$ as a new energy unit, the unit effective output interval $[20,45]$, the incremental power generation income is 100 yuan / MWh.

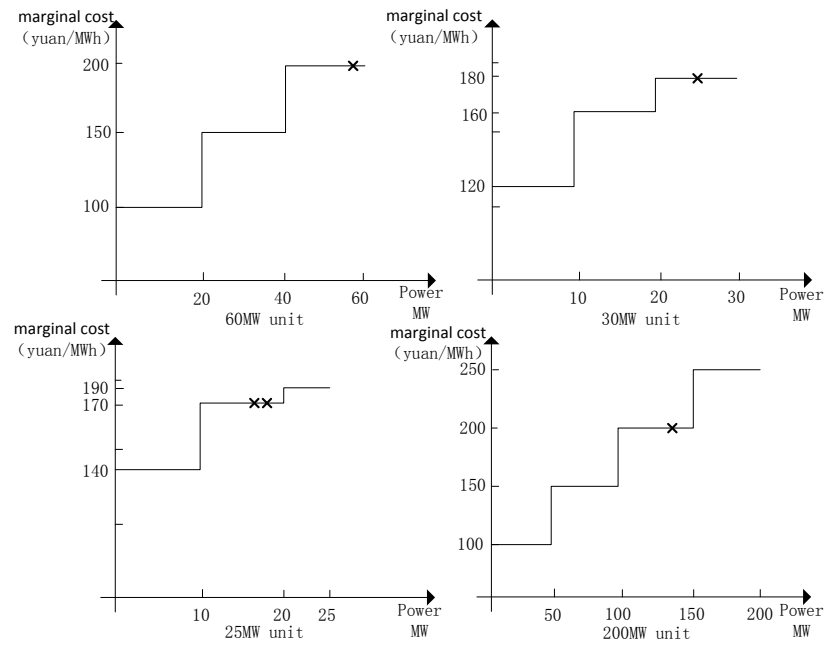

Fig. 5 The marginal cost schematic of different units in different force intervals.

In this paper, the initial population size of the particle swarm algorithm is set to 50, the maximum number of iterations is 100 , the inertia weight $\mathrm{w}=0.729$, and the accelerating factor $c_{1}=c_{2}=1.496$. According to the target optimization model, the load adjustment and the unit output are combined to optimize. The optimal scheme is shown in Table 2, and the net benefit of the whole society is 4685.6 yuan; It can be seen from the optimization results that the optimization process is divided into four steps, in the step by step optimization process, the voltage amplitude and phase angle changes as shown in Figure 6 and Figure 7; incremental change of loss is shown in Table 3.

Table 2. "load-grid-source" collaborative optimization results.

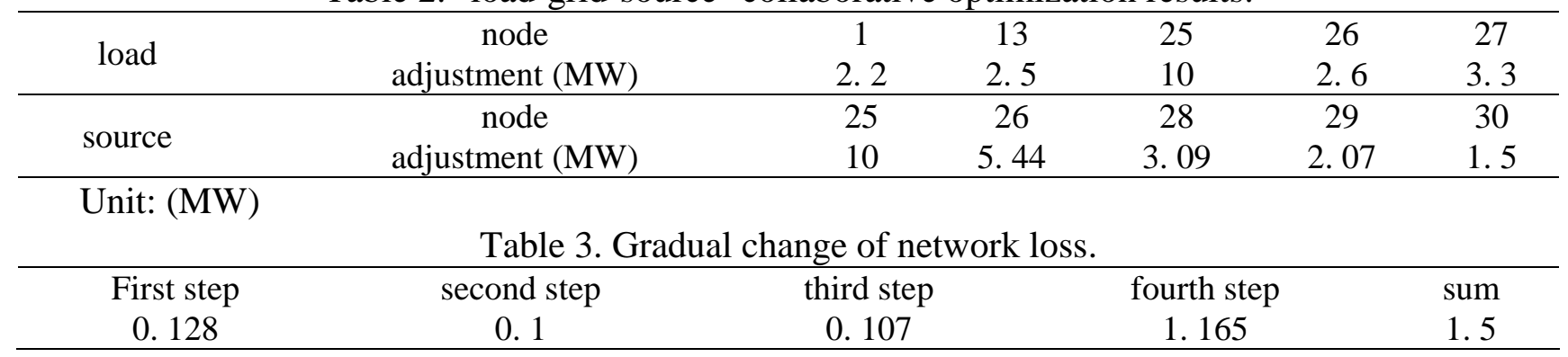




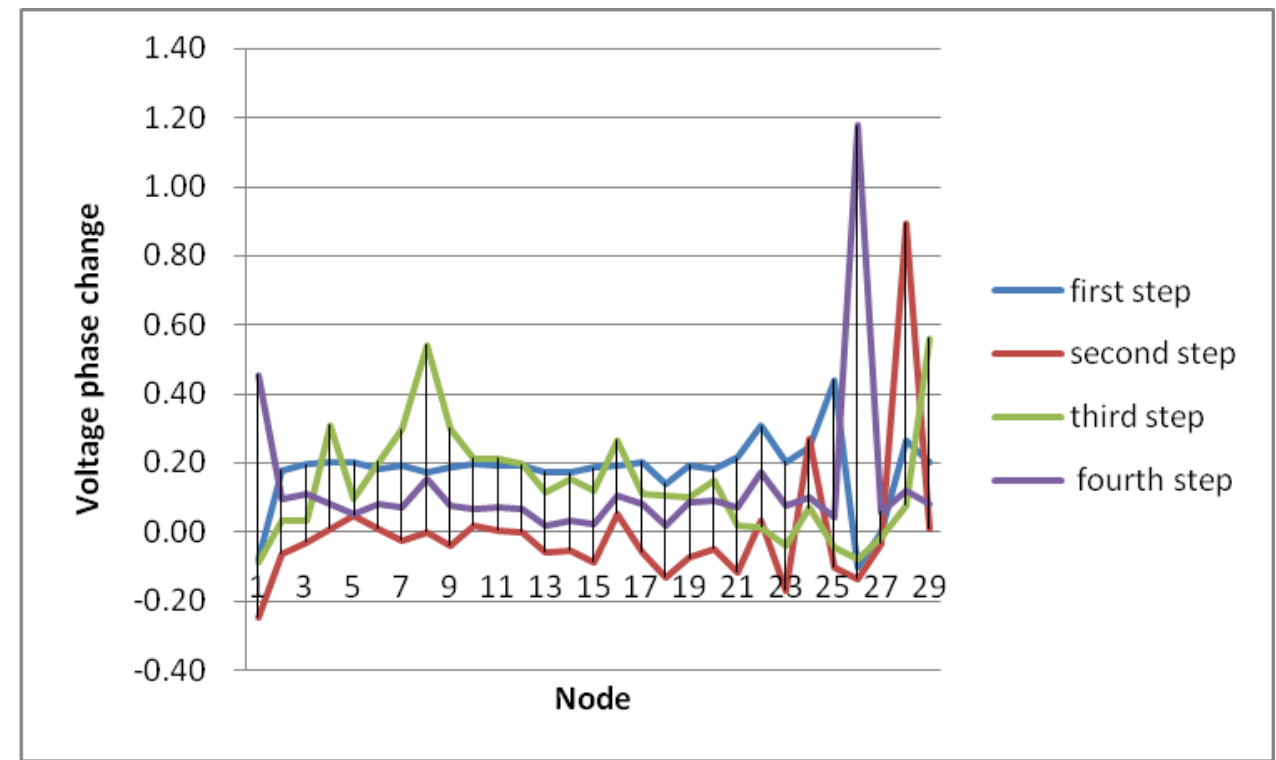

Fig. 6 the phase change of node voltage during the step optimization process.

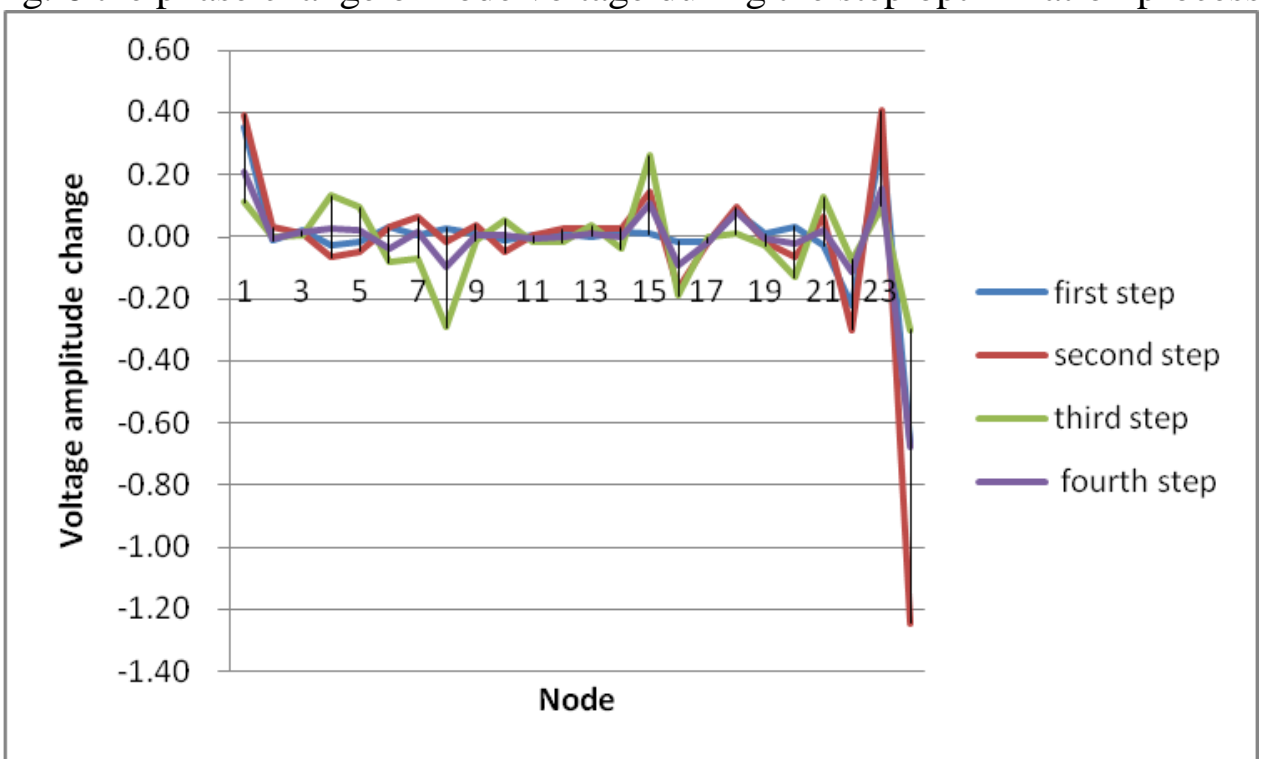

Fig. 7 the change of node voltage amplitude during the step optimization process.

Analysis of the optimization results and we can get the conclusions as follows:

(1). For the heavy-duty area, because of its own load increase will cause a sharp increase in loss, it should be used as a demand response side resource for peak shifting;

(2). From the perspective of society as a whole, only when the marginal revenue of the heavy-duty area exceeds a certain value, the increase in income can compensate for the cost of the source side and the incremental cost of the network loss.

(3). The local consumption of the new energy can reduce the loss caused by power of long-distance transmission;

(4). By digging more adjustable resources that tracking new energy can minimize the amount of abandoned wind power and solar.

\section{Conclusion}

In this paper, through the establishment of "load - grid - source" collaborative optimization mathematical model, taking into account the power grid incremental load on the source and power grid, From the perspective of society as a whole, considering the new energy increment power generation income, load adjustment income, generators increment Power generation costs and power grid incremental loss cost ,then optimizing the incremental load adjustment and power increment 
output to maximize the benefits of the whole society, at the same time, which can provides a theoretical basis for China's power market support services market development.

\section{Reference}

[1]. SHU Yinbiao, ZHANG Zhigang, GUO Jianbo, ZHANG Zhengling, Study on Key Factors and Solution of Renewable Energy Accommodation [J]. Proceedings of the CSEE, 2017, 37 (1):1-8.

[2]. SUN Yi, ZHOU Shuang, SHAN Baoguo, et al. Analysis of Electeic Power Alternative Potential Under Multi-Scenario [J]. Power System Technology, 2017, 41 (1):118-123.

[3]. He Huimin, Wang Haiyan, Ren Jie, et al. Study on Wind Power Accommodation by Using Curtailed Wind Power to Heat for Power Alternative [J]. North China Electric Power, 2016 (1):1-5.

[4]. PARSA M, ABDOLLAHI a, RASHIDINEJAD M. Flexible demand response modeling in competitive electricity markets [J]. Applied Energy, 2011, 88 (9): 3257-3269

[5]. JU Ping, QIN Chuang, HUANG Hua, et al. Research Trends of Power System Modeling Geared to Smart Grid [J]. Automation of Electric Power Systems, 2012, 36 (11):1-6.

[6]. LIU Xiao. Theoretical Study on Wide - area Source - Load Interaction Scheduling Model for New Energy Power System [D]. Beijing: North China Electric Power University, 2012

[7]. SUN Yi, XU Peng, SHAN Baoguo, QI Bing. Road Map for "Internet Plus" Energy Substitution in Electricity Retail Market Reform in China [J]. Power System Technology,2016,40 (12):36483654.

[8]. YAO Jianguo, et al. Model and Strategy for Multi-Time Scale Coordinated Flexible Load Interactive Scheduling [J]. Proceedings of the CSEE: 2014, 34 (22):3664-3673.

[9]. Yao jianguo, Yang shengchun, Wang ke, Yang zhenglin, et al. Concept and research framework of smart grid "source-grid-load" interactive operation and control [J]. Automation of Electric Power Systems, 2012, 36 (12):1-6.

[10]. LI Ming, HU Diangang, ZHOU Youxue, Research and Practice of Renewable Energy Local Consumption Mode in Gansu Province Based on "Double Alternative" Strategy [J]. Power System Technology, 2016, 40 (10):2991-2997.

[11]. Zhang Boming, Chen Shou Sun, Yan Zheng. Analysis of high - power networks [M]. Version 2. - Beijing: Tsinghua University Press, 2007. 9 (2015. 8 reprint). 